\title{
DEPOLARIZATION DISPERSION OF TOTALLY SYMMETRIC FUNDAMENTALS IN RESONANCE RAMAN SPECTRA. ANALYSIS FOR AZOBENZENE DYES
}

\author{
P. SAssi* and M. PaWlikowski \\ Department of Theoretical Chemistry, Jagiellonian University, Faculty of Chemistry \\ Ingardena 3, 30-060 Kraków, Poland
}

(Received February 6, 1996; revised version May 8, 1996)

The resonance Raman excitation profiles and depolarization dispersions for totally symmetric vibrations are studied under the energy region corresponding to an excitation of two low-energy states of 4-nitro- $4^{\prime}$-diethylaminoazobenzene dye. In a contrast to the earlier analysis of this dye we assume that the low-energy states of the molecule studied have different polarizations. It results in the depolarization dispersions of the Raman lines of totally symmetric vibrations. The effect is illustrated for two vibrations: $\nu_{4}=1342 \mathrm{~cm}^{-1}$ and $\nu_{7}=1448 \mathrm{~cm}^{-1}$ for which excitation profiles show distinctive intensity distribution pattems due to interferences between the vibrational manifolds of the overlapping states. The nature of the electronic (resonant) states and the magnitudes of Franck-Condon parameters determined from the experiment are also briefly discussed.

PACS numbers: $33.20 . \mathrm{Fb}$

\section{Introduction}

Electronic excitation of a molecule tends to enhance the totally symmetric progressions in the resonance Raman (RR) spectra. The distribution of intensity in these progressions is governed by the Franck-Condon (FC) overlap integrals resulting from nonorthogonality of the ground and the excited state(s) vibrational functions [1-3]. In the RR scattering, the FC overlap integrals entering the scattering tensor are weighted by the different complex energy denominators. Therefore, the interferences will arise leading to complex behaviors of the Raman intensities viewed as functions of excitation energy of the laser light [3-6]. The interferences become of special importance if two or more electronic dipole-allowed (resonant)

* On leave from Dipartimento di Chimica, Universita di Perugia, Italy. 
states of different polarization differ by a small energy gap, i.e., when the vibrational manifolds of electronic states overlap. In such a case, the interferences affect not only intensity distribution in the excitation profiles (EPs) but also the depolarization dispersions (DDs) of the Raman transitions.

The situation like that described above may arise when dealing with $R R$ spectra of azobenzene dyes [7-11]. In the low-energy region of these molecules, the absorption spectra reveal two bands associated with dipole-allowed $\Phi_{m}$ and $\Phi_{n}$ states separated only by a few hundred wave numbers depending on the dye at hand [7]. These two states can be identified as a mixture of the charge transfer, $n \pi^{*}$ and $\pi \pi^{*}$ states. The mixed character of these states and the low symmetry of the azobenzene dyes cause that the dipole moments of $\Phi_{0} \rightarrow \Phi_{m}$ and $\Phi_{0} \rightarrow \Phi_{n}$ transitions are unlikely to be parallel. A proximity of $\Phi_{m}$ and $\Phi_{n}$ states, on the one hand, and their different polarizations, on the other hand, allow us to suspect that the considerable DDs will arise when exciting azobenzene dyes in their low-energy absorption region. Unfortunately, nothing is known about the polarization of transition dipole moments in azobenzene dyes. For that reason, the DDs -problem was eliminated in the earlier theoretical studies of azobenzene dyes $[7,8]$. - More specifically it was assumed that both $\Phi_{0} \rightarrow \Phi_{m}$ and $\Phi_{0} \rightarrow \Phi_{n}$ transitions have the same polarization.

- In this paper we aim to discuss in some detail the RR excitation profiles and depolarization dispersion of the fundamental transitions of totally symmetric vibrations of the 4-nitro-4'-diethylaminoazobenzene (DAB) dye taken as an illustrative example. To keep a close relevance to the previous studies concerning azobenzene dyes we retain the principle assumptions made in the earlier treatments [8], but we shall relax the assumption that the dipole moments for the $\Phi_{0} \rightarrow \Phi_{m}$ and $\Phi_{0} \rightarrow \Phi_{n}$ transitions are of the same polarization. In the interest of brevity we restrict our discussion to the fundamental transitions leaving aside the vibronic coupling problems which may result since the $\Phi_{m}$ and $\Phi_{n}$ states are close on the energy scale. An extension of the model to encompass the vibronic coupling problems is straightforward on the formal basis but it would introduce additional model parameters unable to be extracted from the currently available RR data for azobenzene dyes. Perhaps an advent of new RR experimental data concerning certain azobenzene dyes [11] will claim to go beyond the simple model applied in this study.

\section{Model}

According to time-independent theory of the resonance Raman phenomenon, the laser light with an energy $\Omega$ induces the vibrational transitions $\Psi_{0,0} \rightarrow \Psi_{0, k}$ characterized by the tensor [2]:

$$
\alpha_{\sigma, \rho}(0 \rightarrow k)=\sum_{s} \sum_{v_{1}=0}^{\infty} \sum_{v_{2}-0}^{\infty} \ldots \frac{\left(\left\langle\Psi_{0, k}\left|D_{\sigma}\right| \Psi_{s, v}\right\rangle\right)\left(\left\langle\Psi_{s, v}\left|D_{\rho}\right| \Psi_{0,0}\right\rangle\right)}{E_{s, v}-E_{0,0}-\mathrm{i} \Gamma_{s, v}-\Omega}
$$

$\Psi_{0,0}, \Psi_{0, k}$ and $\Psi_{s, v}$ represent, respectively, the initial, the final and the intermediate states of a molecule and $\Gamma_{s, v}$ is the line width describing homogeneous broadening through radiative and (predominately) non-radiative damping. The vectors 
$0=\left(0_{1}, 0_{2}, \ldots\right), k=\left(k_{1}, k_{2}, \ldots\right)$ and $v=\left(v_{1}, v_{2}, \ldots\right)$ abbreviate the sets of vibrational quantum numbers in the states involved in the scattering process.

To use Eq. (1) in a practical way, we will assume that the Born-Oppenheimer approach provides an appropriate, even if approximate first step to describe the electronic states of a molecule. Further, let us assume that Condon approximation is sufficiently accurate to describe the ground-excited state (vibronic) transitions. At this level, the transition dipole moments in Eq. (1) can be evaluated from

$$
\begin{aligned}
& \left(\left\langle\Psi_{0, k}|D| \Psi_{s, v}\right\rangle\right)=D_{0, s}(0)\left(\xi_{0, k} \mid \xi_{s, v}\right), \\
& \left(\left\langle\Psi_{s, v}|D| \Psi_{0,0}\right\rangle\right)=D_{0, s}(0)\left(\xi_{s, v} \mid \xi_{0,0}\right),
\end{aligned}
$$

where $\Phi_{0}$ and $\Phi_{s}$ (for $s=m, n$ ) are the ground state and two excited state wave functions. $\xi_{0, k}(Q)$ and $\xi_{s, v}(Q)$ are the corresponding vibrational functions.

Further we will assume that the vibrational manifolds are described by $N$ harmonic oscillators represented by the set of mass-weighted normal coordinates $Q$. These oscillators are displaced but they are neither distorted nor rotated relative to these in the ground state. It means that the effects due to changes of vibrational frequencies upon electronic excitation and Dushinsky rotations $[5,15]$ are not interesting for us in this paper. At this level of approximation, the FC overlap integrals $\left(\xi_{0, k} \mid \xi_{s, v}\right)$ and $\left(\xi_{s, v} \mid \xi_{0, k}\right)$ have simple analytical forms determinable from well-known recursion formulas [3]. Therefore, the products $\left(\xi_{0, k} \mid \xi_{s, v}\right)\left(\xi_{s, v} \mid \xi_{0,0}\right)$ for the fundamental transitions characterized by vectors $k=\left(0_{1}, 0_{2}, \ldots 1_{i}, \ldots\right)$ and the energy differences $E_{s, v}-E_{0,0}$ in Eq. (1) can be calculated from

$$
\begin{aligned}
& \left(\xi_{0,1} \mid \xi_{s, v}\right)\left(\xi_{s, v} \mid \xi_{0,0}\right)=X_{s, i}^{1}\left(v_{i}-X_{s, i}^{2}\right) \prod_{j=1}^{N} \frac{1}{v_{j} !}\left(X_{s, j}^{2}\right)^{v_{j}} \exp \left(-X_{s, j}^{2}\right), \\
& E_{s, v}=E_{0,0}-E_{s}(0)-E_{0}(0)+\sum_{i=1}^{N} \omega_{i}\left(-X_{s, i}^{2}+v_{i}\right),
\end{aligned}
$$

where $X_{s, j}=B_{s, j} / \sqrt{2} . B_{s, i}$ is dimensionless FC parameter of the $i$-th vibration in the $s$-th electronic state. $E_{0}(0)$ and $E_{s}(0)$ are the electronic potential energies of the corresponding states taken in the ground state nuclear equilibrium configuration at $Q=0$.

To proceed further we have to address the problem of the forms which should be given for the transition dipole moments $D_{0, m}(0)$ and $D_{0, n}(0)$ in Eq. (1). To our best knowledge nothing is known about polarizations of the ground-excited state transitions in azobenzene dyes. However, if such transition dipole moments would be known, e.g., from quantum chemical calculations we could always decompose them as

$$
\begin{aligned}
& D_{0, m}(0)=D_{m}\left(e_{x} \cos \varphi+O e_{y}+e_{z} \sin \varphi\right), \\
& D_{0, n}(0)=D_{n}\left(e_{x} \cos \varphi+O e_{y}-e_{z} \sin \varphi\right),
\end{aligned}
$$

where $D_{m}$ and $D_{n}$ are the "lengths" of transition dipole moments and $2 \varphi$ is an angle between them. Therefore, inserting (4a)-(4b) into (2a)-(2b) and then results 
to Eq. (1) we express the scattering tensor in the compact form as

$$
\begin{aligned}
& \{\alpha\}_{0}^{k}=\frac{1}{2}\left(\begin{array}{lll}
R_{+}, & 0, & 0 \\
0, & 0, & 0 \\
0, & 0, & R_{+}
\end{array}\right)+\frac{1}{2}\left(\begin{array}{ccc}
R_{+}, & 0, & 0 \\
0, & 0, & 0 \\
0, & 0, & -R_{+}
\end{array}\right) \cos 2 \varphi \\
& +\frac{1}{2}\left(\begin{array}{ccc}
0, & 0, & R \\
0, & 0, & 0 \\
R_{-}, & 0, & 0
\end{array}\right) \sin 2 \varphi
\end{aligned}
$$

where $R_{+}$and $R_{-}$are defined as

$$
R_{ \pm}=\sum_{\boldsymbol{v}}\left[\frac{\left(\xi_{0, k} \mid \xi_{m, v}\right) \mid\left(\xi_{m, v} \mid \xi_{0,0}\right)}{E_{m, \boldsymbol{v}}-E_{0,0}-\Omega-\mathrm{i} \Gamma_{m, v}} D_{m}^{2} \pm \frac{\left(\xi_{0, k} \mid \xi_{n, v}\right) \mid\left(\xi_{n, v} \mid \xi_{0,0}\right)}{E_{n, \boldsymbol{v}}-E_{0,0}-\Omega-\mathrm{i} \Gamma_{n, \boldsymbol{v}}} D_{n}^{2}\right]
$$

Equation (5) shows that for $\varphi \neq 0$ the fundamental transitions are governed by the symmetric isotropic and symmetric anisotropic type of scattering and the anisotropic symmetric scattering becomes not active when $\varphi$ vanishes. It should be noted that the anisotropic antisymetric scattering does not operate for all Raman transitions discussed in this paper. This is a consequence of the assumption that the $\Phi_{m}$ and $\Phi_{n}$ states are not vibronically coupled through the totally symmetric vibration(s). Thus the depolarization ratio obtainable from Eq. (3) of Ref. [2]

$$
\rho(\Omega)=\frac{3 \sin ^{2} 2 \varphi+\left(1+3 \cos ^{2} 2 \varphi\right)\left|R_{+} / R_{-}\right|^{2}}{4 \sin ^{2} 2 \varphi+4\left(2+\cos ^{2} 2 \varphi\right)\left|R_{+} / R_{-}\right|^{2}}
$$

is allowed to vary in the range $[0,3 / 4] ; \rho$ being equal to $1 / 3$ when the transition dipole moments are parallel $(\varphi=0)$.

The excitation profiles presented in the next section were calculated from the expression

$$
\Theta_{i}(\Omega) \propto\left|R_{+}\right|^{2}\left(9+7 \cos ^{2} 2 \varphi\right)+7\left|R_{-}\right|^{2} \sin ^{2} 2 \varphi,
$$

which can be readily obtained from the tensor (5) and Eqs. (2), (4) and (5) of Ref. [2]. The Raman intensities of the particular lines were calculated as products of $\Theta_{i}$ and the characteristic $\Omega\left(\Omega-\omega_{i}\right)^{3}$ factors. In all calculations we assumed the same line width in all vibrational levels of the two states. The inhomogeneous broadening $(\Delta)$ due to interaction between the molecule and solvent $\left(\mathrm{CCl}_{4}\right)$ was included in the standard well-known fashion $[2,8]$.

\section{Results and discussion}

Of many dyes derivable from trans-azobenzene parent molecule, those consisting of the $-\mathrm{NO}_{2}$ electron-withdrawing and $-\mathrm{N}(\mathrm{R})_{2}$ electron-donating groups have been studied in the series of recent papers [7-11]. The electronic structures of these dyes do not change much when going from one dye to another. Therefore, for purpose of this paper we select 4-nitro-4'-diethylaminoazobenzene dye as a representative of whole family of azobenzene dyes.

The low-energy part of the absorption spectrum of $\mathrm{DAB}$ molecule shows two broad structureless bands $\left(\Gamma=800 \mathrm{~cm}^{-1}\right)$ with the maxima at $20419 \mathrm{~cm}^{-1}$ 
and $21460 \mathrm{~cm}^{-1}$ and with the intensity ratio estimated to be $\left|D_{m} / D_{n}\right|^{2} \approx 1.4$. These maxima correspond roughly to the origins of $\Phi_{0} \rightarrow \Phi_{m}$ and $\Phi_{0} \rightarrow \Phi_{n}$ transitions. The lack of vibrational structures in the absorption bands did not allow us to extract the FC parameters directly from absorption spectra. Thus, the pairs $\left(B_{m, i}, B_{n, i}\right)$ of FC parameters were obtained in such a way to achieve the best reconstruction for the eight excitation profiles measured by one of us (P.S.) for the most active totally symmetric vibrations of $\mathrm{DAB}$ dye. The $\mathrm{FC}$ parameters obtained in that way agree very well with those reported earlier [8] for the five totally symmetric vibrations of DAB dye. The sets $\left\{B_{m, i}\right\}$ and $\left\{B_{n, i}\right\}$ could then be used to reconstruct the experimental RR spectra in the fundamental and overtone regions of Raman transitions. The sample of results for the laser excitation energy at $20419 \mathrm{~cm}^{-1}$ is shown in Figs. 1a and 1b. The model parameters are collected in Table together with the assignments of eight totally symmetric vibrations taken in the calculations. The angle $\varphi$ was taken somewhat arbitrarily to be $30^{\circ}$.

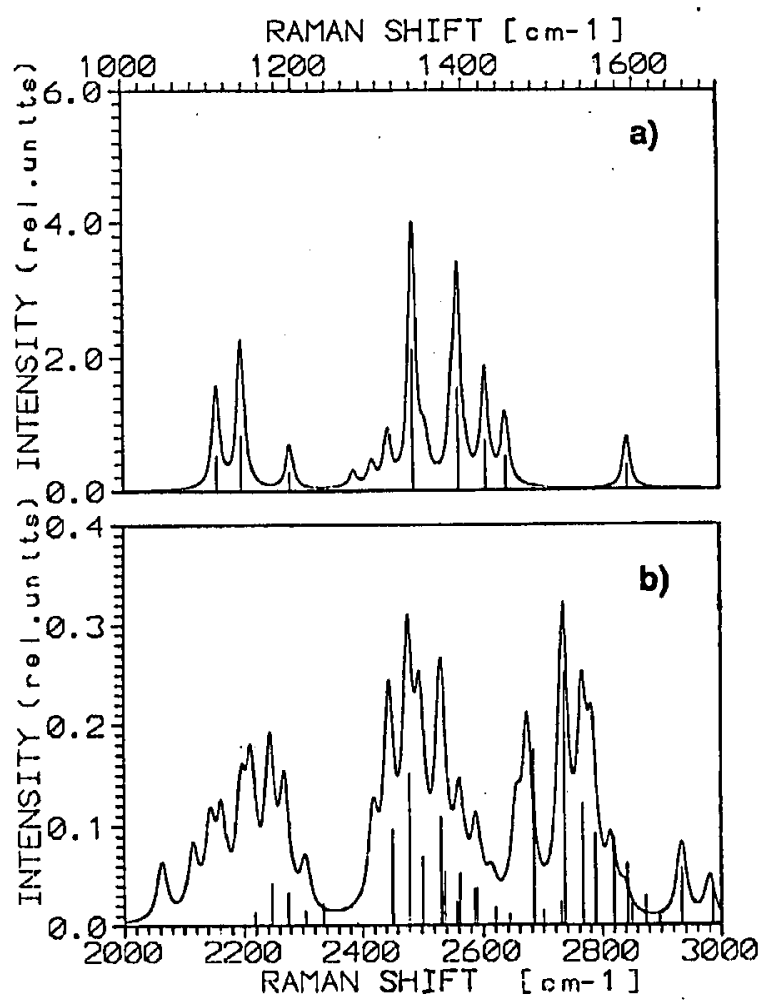

Fig. 1. The measured (curve) and calculated (sticks) RR spectrum of 4-nitro-4'-diethylaminoazobenzene dye for $\Omega=20419 \mathrm{~cm}^{-1}$; (a) the fundamental region, (b) the overtone region. The intensity scale is relative but the scaling factor is the same for both (a) and (b) graphs. 
TABLE The model parameters applied to reconstruct the RR experimental data for 4-nitro-4'-diethylamino azobenzene dye.

\begin{tabular}{c|r|r|l}
\hline \hline Frequency $\nu_{i}\left[\mathrm{~cm}^{-1}\right]$ & $B_{m, i}$ & $B_{n, i}$ & \multicolumn{1}{c}{ Assignment } \\
\hline$\nu_{1}=1109$ & 0.29 & 0.01 & $\delta(\mathrm{C}-\mathrm{II})$ deformation \\
$\nu_{2}=1138$ & 0.36 & 0.02 & $\delta(\mathrm{C}-\mathrm{II})$ deformation \\
$\nu_{3}=1196$ & 0.21 & 0.14 & $\delta(\mathrm{C}-\mathrm{II})$ deformation \\
$\nu_{4}=1342$ & 0.53 & -0.05 & sym. stretch $-\mathrm{NO}_{2}$ \\
$\nu_{5}=1394$ & 0.45 & -0.02 & stretching $-\mathrm{N}=\mathrm{N}-$ \\
$\nu_{6}=1425$ & 0.31 & -0.01 & ring stretching \\
$\nu_{7}=1448$ & 0.26 & 0.16 & ring stretching \\
$\nu_{8}=1591$ & 0.21 & 0.14 & ring stretching \\
\hline \\
$D_{n} / D_{m 2}=1.2, E_{m}(0)=21000 \mathrm{~cm}^{-1}, E_{n}(0)=21500 \mathrm{~cm}^{-1}$, \\
$\Gamma_{z, v}=\Gamma=500 \mathrm{~cm}^{-1}$ and $\Delta=30 \mathrm{~cm}^{-1}$ for all $s$ and $v$.
\end{tabular}

From Fig. 1a one can see that the agreement with the experiment is in general satisfactory for all fundamental lines. Note that only slightly worse results could be achieved for the overtones and combination tones as showed in Fig. $1 \mathrm{~b}$. An assignment of the weak features at $1271 \mathrm{~cm}^{-1}, 1293 \mathrm{~cm}^{-1}$ and $1312 \mathrm{~cm}^{-1}$ not reproduced in our model is not certain. Most probably they correspond to the fundamental excitations of $\mathrm{C}-\mathrm{II}$ deformation and $\mathrm{Ph}-\mathrm{N}=$ motions involved in an appreciable mixing as it was indicated elsewhere [12-14]. Because of that mode mixing, the quantitative interpretation of these Raman lines is beyond the theoretical model applied in this study. The most intense fundamental transitions at $1342 \mathrm{~cm}^{-1}$ and $1394 \mathrm{~cm}^{-1}$ could be assigned as the symmetric stretch vibrations of $-\mathrm{NO}_{2}$ and $-\mathrm{N}=\mathrm{N}-$ groups, respectively. Prominent activity of $-\mathrm{NO}_{2}$ stretch mode seems to reflect considerable rearrangement of electron density in the $-\mathrm{NO}_{2}$ group due to a withdrawal of electrons from the benzene rings. In fact this effect is responsible for the shift of electronic energy levels resulting in changes of the color of the azobenzene dyes. Because the rearrangement of the electrons due to substituents affects the electron density of the benzene rings, it is conceivable that symmetric stretcling motions of $-\mathrm{N}=\mathrm{N}-$ and $-\mathrm{C}=\mathrm{C}-$ groups will show substantial FC activity in the $\Phi_{m}$ state as evidenced in the RR experiment. The different situation should occur in the $\Phi_{n}$ state which reveals predominately the bipolar charge transfer character with a small admixture of $n \pi^{*}$ and $\pi \pi^{*}$ states. In a case like that, both ring stretching and deformation motions of the benzene rings are expected to be primarily $\mathrm{FC}$ active. This qualitative picture is consistent with the sizes of FC parameters listed in Table and, by implication, with the RR experimental data studied in this paper. It is interesting to note that FC parameters in the $\Phi_{n}$ state are much smaller than those in the $\Phi_{m}$ state for every totally symmetric vibrations studied. Moreover, some of the FC parameters are of opposite signs indicating an increase and decrease in bond lengths in the excited states relative to the ground state. Such a situation favors the interferences which lead to distinctive shapes of the EPs depending on whether $B_{m, i} B_{n, i}<0$ or $B_{m, i} B_{n, i}>0$ holds for the vibra- 
tion in question. Examples are given in Fig. 2 and Fig. 3 for two $\nu_{4}=1342 \mathrm{~cm}^{-1}$ and $\nu_{7}=1448 \mathrm{~cm}^{-1}$ vibrations. The dashed, dotted and solid lines were calculated for $\varphi=5^{\circ}, \varphi=15^{\circ}$ and $\varphi=30^{\circ}$, respectively. The experimental intensities are marked as dark circles. These were recently remeasured [11] with a use of better technical equipment than that applied previously [7]. The ordinates of both figures are given in relative units but the scaling factor is the same for both $\nu_{4}$ and $\nu_{7}$ vibrations.

From Fig. 2 and Fig. 3 one can see that overall agreement between theory and experiment is very good. While the EP calculated for $\nu_{4}$ vibration only slightly depends on the angle $\varphi$, the solid lines match the experimental EPs much better than the dashed and dotted lines do, especially for $\nu_{7}$ vibration. A systematic improvement between the theory and the experiment as showed for $\nu_{7}$ vibration could be achieved for all vibrations from Table for which $B_{m, i} B_{n, i}>0$. This result rationalizes the choice of $\varphi$ used to calculate the RR spectrum in Fig. 1. It also suggests that the transition dipole moments to $\Phi_{m}$ and $\Phi_{n}$ states are not of the same orientation as it was assumed elsewhere [8]. However we wish to emphasize that the information derived from the EPs analysis alone are not sufficient to assess the angle $\varphi$ with a required accuracy. Namely, one can argue that the effect showed in Fig. 3 is in part due to interferences between the vibrational manifolds of $\Phi_{m}$

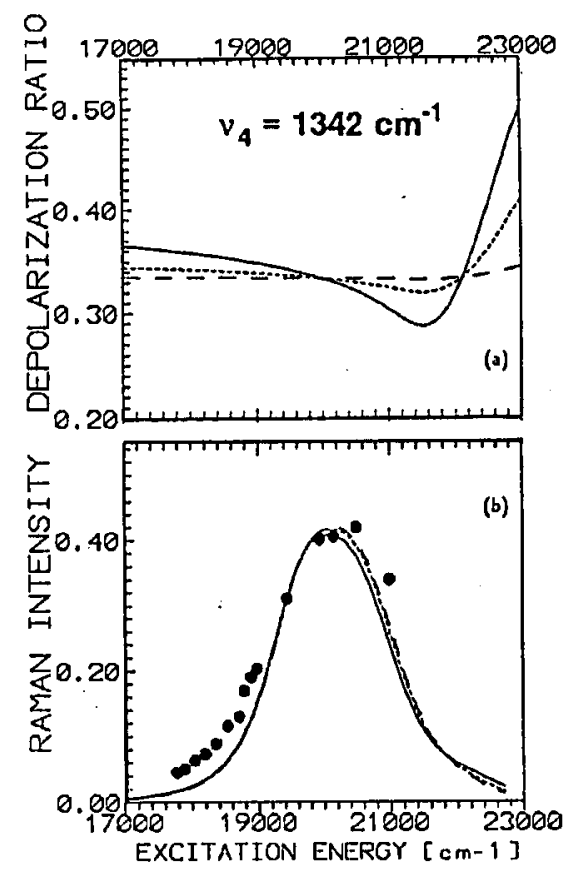

Fig. 2. The EP (b) and DD (a) of the $\nu_{4}$ vibration aclieved for the different angle: $\varphi=5^{\circ}$ (dashed line), $\varphi=15^{\circ}$ (dotted line) and $\varphi=30^{\circ}$ (solid line). The dark circles are the experimental data. 

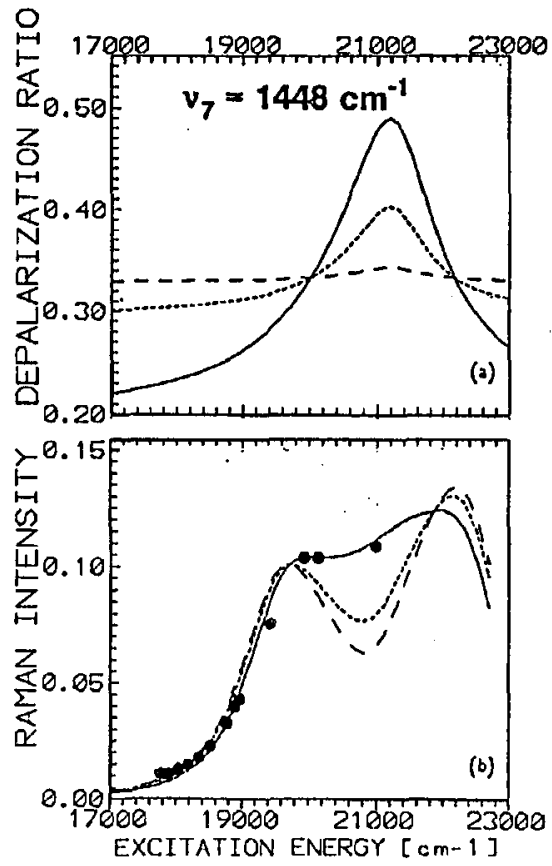

Fig. 3. The same plots as Fig. 2 but for the $\nu_{7}$ vibration.

and $\Phi_{n}$ states and one or more high-energy off-resonance state(s) not included in the present model. Such interferences are common features of the RR phenomenon [16] and may modify the DDs curves presented in Figs. 2 and 3. Since the energy difference between $\Phi_{m}$ (or $\Phi_{n}$ ) state and an ligh-energy state varies in a range 10000-12000 $\mathrm{cm}^{-1}[8]$, an influence of the off-resonance state(s) on the DDs is not expected to be large in the case discussed in this paper. In this view, however, there are no doubts that more experimental and theoretical studies are needed to proceed with a solution of problem concerning paper.

In general case the DD curves may show complex behaviors depending on the energy separation of the overlapping states, their polarizations and the sizes and signs of FC parameters in the resonant states. It is so due to interferences manifested not only in the resonance but also in the pre-resonance excitation region of the $\Phi_{m}$ state. To gain an insight into DDs behaviors in the pre-resonance region, let us assume that a vibration with the normal coordinate $Q_{i}$ is a subject to small FC displacements in both $\Phi_{m}$ and $\Phi_{n}$ states and let the pre-resonance condition $\Omega<E_{m}(0)$ be satisfied. Because of inequalities $\left|B_{m, i}\right| \ll 1,\left|B_{n, i}\right| \ll 1$ assumed, we can retain only linear terms in $B$ in $\mathrm{Eq}$. (3a). Therefore, the ratio $R_{+} / R_{-}$needed to calculate the depolarization ratio in the pre-resonance region can be evaluated from

$\frac{R_{+}}{R_{-}}=\frac{\left[E_{m}(0)-\Omega\right]\left(B_{m, i} D_{m}^{2}+B_{n, i} D_{n}^{2}\right)-2\left[E_{n}(0)-E_{m}(0)\right] B_{n, i} D_{n}^{2}}{\left[E_{m}(0)-\Omega\right]\left(B_{m, i} D_{m}^{2}-B_{n, i} D_{n}^{2}\right)+2\left[E_{n}(0)-E_{m}(0)\right] B_{n, i} D_{n}^{2}}$. 
For $B_{m, i} B_{n, i}>0$, a closer analysis of Eq. (8) reveals that the ratio $\left|R_{+} / R_{-}\right|$ will decrease when the laser energy departs from the resonance with the $\Phi_{m}$ state. In this case, the depolarization ratio will tend to the value of $3 / 4$. The opposite holds for $B_{m, i} B_{n, i}<0$. Now, the ratio $\left|R_{+} / R_{-}\right|$increases as the excitation energy $\Omega$ drops. Therefore, the depolarization ratio tends to the value of $0.25\left(1+\cos ^{2} 2 \varphi\right) /\left(1+3 \cos ^{2} 2 \varphi\right)$ which strongly depends on the transition dipole moment orientations. Such trends are just observed in Fig. 2 and Fig. 3 for $\nu_{4}$ and $\nu_{7}$ vibrations of $\mathrm{DAB}$ dye. Unfortunately, so far available RR experimental data did not allow us to verify the actual DDs results for DAB molecule. Therefore they have to remain as the theoretical predictions hopefully verifiable by the new measurements of $R R$ spectra for 4-nitro-4'-diethylaminoazobenzene dye, in particular, and other azobenzene dyes, in general [11].

\section{Conclusion}

We have studied the $R R$ characteristics of the 4-nitro-4'-diethylaminoazobenzene molecule considered as a representative example of whole family of azobenzene dyes. In contrast to earlier treatment in this field [8], the formulated theoretical model allows the low-energy (resonant) states of azobenzene dyes to be of different polarizations. It leads to DDs of Raman transitions not studied earlier. The basic functionality of the model was demonstrated for the eight fundamental transitions for which the calculated Raman spectrum agrees excellently well with the spectrum recently remeasured for the DAB dye [11]. However, due to an absence of relevant experimental data, the DDs calculated for the Raman transitions studied have to remain at the level of "theoretical predictions" at present time. The preliminary DDs study for the azobenzene dyes [11] indicates that the depolarization ratios measured for selective excitation energies do not exceed a value of $\rho=3 / 4$. It suggests that the anisotropic antisymmetric components of the scattering tensor should not contribute much to the scattering for all totally symmetric vibrations studied in this paper. It allows us to conclude that the vibronic coupling effects abandoned by us in this paper should not be large. As the works undertaken by one of us (P. Sassi) are in progress we believe that the theoretical predictions regarding DDs presented in this paper will be verified soon by the RR experiments.

\section{References}

[1] J. Tang, A.C. Albrecht, in: Raman Spectroscopy - Theory and Practice, Ed. H.A. Szymanski, Vol. II, Plenum, New York 1970, Ch. 2.

[2] W. Siebrand, M.Z. Zgierski, in: Excited States, Ed. E.C. Lim, Vol. IV, Academic, New York 1979, Ch. 2.

[3] W. Siebrand, M.Z. Zgierski, J. Chem. Phys. 71, 3561 (1979).

[4] M. Pawlikowski, Acta Phys. Pol. A 63, 547 (1983).

[5] K. Zborowski, M. Pawlikowski, Acta Phys. Pol. A 88, 435 (1995).

[6] W.H. Henneker, W. Siebrand, M.Z. Zgierski, J. Chem. Phys. 74, 6560 (1981).

[7] R.S. Catalliotti, S.M. Murgia, G. Paliani, A. Poletti, M.Z. Zgierski, J. Raman Spectrosc. 10, 251 (1985). 
[8] R.S. Catalliotti, S.M. Murgia, G. Paliani, A. Poletti, M.Z. Zgierski, J. Raman Spectrosc. 10, 258 (1985).

[9] A. Morresi, G. Paliani, S. Santini, R.S. Catalliotti, Can. J. Speclrosc. 33, 69 (1988).

[10] R.S. Catalliotti, A. Morresi, G. Paliani, M.Z. Zgierski, J. Raman Spectrosc. 20, 601 (1989).

[11] P. Sassi, to be published.

[12] T. Uno, B.K. Kim, Y. Saito, K. Machida, Spectrochem. Acta A 32, 1179 (1976).

[13] K. Kumar, P.R. Carey, Can. J. Chem. 55, 1444 (1977).

[14] T. Uno, H. Lee, Y. Saito, K. Machida, Spectrochem. Acta A 32, 1319 (1976).

[15] W.H. Henneker, M. Pawlikowski, W. Siebrand, M.Z. Zgierski, J. Phys. Chem. 87, 4805 (1985).

[16] M. Zgierski, J. Raman Spectrosc. c, 53 (1977). 